\title{
MODULI OF VECTOR BUNDLES ON CURVES WITH PARABOLIC STRUCTURES
}

\author{
BY C. S. SESHADRI
}

Communicated by Stephen S. Shatz, April 8, 1976

Let $H$ be the upper half plane and $\Gamma$ a discrete subgroup of Aut $H$. Suppose that $H \bmod \Gamma$ is of finite measure. This work stems from the question whether there is an algebraic interpretation for the moduli of unitary representations of $\Gamma$ similar to the case when $H \bmod \Gamma$ is compact (cf. [3] , [4] , [5]). We show that this is indeed the case via the moduli of vector bundles on the compactification of $H \bmod \Gamma$, provided with some additional structures which we propose to call parabolic structures. The idea of parabolic structures is inspired from A. Weil's work [6, $\$ 2$, Chapter I, p. 56] .

Let $X$ be a smooth, irreducible, projective curve defined, say, over an algebraically closed field $k$. By vector bundles on $X$ we understand algebraic vector bundles.

Definition 1. Let $V$ be a vector bundle on $X$ and $Q \in X$. Then a quasiparabolic structure of $V$ at $Q$ is giving a flag on the fibre $V_{Q}$ of $V$ at $Q$, i.e., giving linear subspaces $F^{i} V_{Q}$ of $V_{Q}$,

$V_{Q}=F^{1} V_{Q} \supset F^{2} V_{Q} \supset \cdots \supset F^{r} V_{Q} ; \quad \operatorname{dim} F^{i} V_{Q}=l_{i} ; \quad l_{1}>l_{2}>\cdots>l_{r}$

We call $l=\left(l_{1}, \ldots, l_{r}\right)$ the type (or flag type) of the quasi-parabolic structure. Let $k_{1}=l_{1}-l_{2}, k_{2}=l_{2}-l_{3}, \ldots, k_{r-1}=l_{r-1}-l_{r}, k_{r}=l_{r}$; then $k_{i}$ are called the multiplicities of the quasi-parabolic structure.

Definition 2. Let $V$ be a vector bundle on $X$ and $Q \in X$. Then a parabolic structure of $V$ at $Q$ is giving

(i) a quasi-parabolic structure of $V$ at $Q$; say $l=\left(l_{1}, \ldots, l_{r}\right)$ is its type and $\left\{k_{i}\right\}$ its multiplicities, and

(ii) constants $\alpha=\left(\alpha_{1}, \ldots, \alpha_{n}\right)$ called the weights of the parabolic structure such that $0 \leqslant \alpha_{1} \leqslant \alpha_{2} \leqslant \cdots \leqslant \alpha_{n}<1$ and there are $r$ distinct elements among $\alpha$, say $\alpha^{\prime}=\left(\alpha_{1}^{\prime}, \ldots, \alpha_{r}^{\prime}\right), 0 \leqslant \alpha_{1}^{\prime}<\alpha_{2}^{\prime}<\cdots<\alpha_{r}^{\prime}<1$, such that $\alpha_{1}^{\prime}$ occurs $k_{1}$ times, $\alpha_{2}^{\prime}$ occurs $k_{2}$ times, . . , $\alpha_{r}^{\prime}$ occurs $k_{r}$ times among $\alpha$. We call $\alpha_{i}^{\prime}$ the weight of $F^{i} V_{Q}$. Note that $l_{1}=n=r k V$.

Let $V, W$ be vector bundles on $X$ with quasi-parabolic structures at $Q$. An isomorphism $f: V \rightarrow W$ of vector bundles is said to be a quasi-parabloic isomorphism if the types of $V, W$ at $Q$ are the same and $f_{Q}\left(F^{i} V_{Q}\right)=F^{i} W_{Q}\left(f_{Q}\right.$ :

AMS (MOS) subject classifications (1970). Primary 14H20, 14H99; Secondary $32 \mathrm{G} 13$. 
isomorphism induced by $f$ on the fibers of $V, W$ at $Q$ ). Suppose, moreover, we are given parabolic structures of $V, W$ at $Q$ consistent with the given quasiparabolic structures; we say that $f$ is a parabolic isomorphism if $f$ is a quasiparabolic isomorphism and weight of $F^{i} V_{Q}=$ weight of $F^{i} W_{Q}$.

Definition 3. Let $V$ be as in Definition 2. Then the parabolic degree of $V$ is defined by

$$
\text { par } \operatorname{deg} V=\operatorname{deg} V+\sum_{i=1}^{n} \alpha_{i} \quad(\operatorname{deg} V=\operatorname{degree} V) .
$$

Also we write $\operatorname{par} \mu(V)$ for the expression

$$
\operatorname{par} \mu(V)=\mu(V)+\left(\sum \alpha_{i}\right) / r k V ; \mu(V)=(\operatorname{deg} V) / r k V .
$$

We give similar definitions when we are given parabolic structures at a finite number of poinis of $X$.

Definition 4. Let $W, V$ be vector bundles on $X$ with parabolic structures at $Q \in X$. We say that $W$ is a parabolic subbundle of $V$ if

(i) $W$ is a subbundle of $V$ in the usual sense;

(ii) given $i_{0}, F^{i_{0}} W \subset F^{j} V$ for some $j$. Let $j_{0}$ be such that $F^{i_{0}} W \subset F^{j_{0}} V$ and $F^{i_{0}} W \not \subset F^{j_{0}+1} V$; then weight of $F^{j_{0}} V=$ weight of $F^{i_{0}} W$.

We define similarly the notion of a parabolic quotient bundle of $V$. Note that given an ordinary subbundle $W$ of $V$ (resp. quotient bundle), there exists a canonical structure of a parabolic subbundle (resp. quotient bundle) on $W$. Following Mumford (cf. [1]), we introduce

Definition 5. Let $V$ be a vector bundle on $X$ with parabolic structures at a finite number of points of $X$. We say that $V$ is parabolic stable (resp. semistable) if $\forall$ proper parabolic subbundle $W$ of $V$, we have $\operatorname{par} \mu(W)<\operatorname{par} \mu(V)$ (resp. $\lessgtr$ ).

Proposition 1. Let $V$ be a vector bundle on $X$ with parabolic structures at a finite number of points of $X$. Suppose that $V$ is parabolic semistable. Then $\exists$ a filtration of $V$ by parabolic subbundles $V_{i}, V=V_{1} \supset V_{2} \supset \cdots$, such that

(i) $\operatorname{par} \mu\left(V_{i}\right)=\operatorname{par} \mu(V)$ and $V_{i}$ is parabolic semistable,

(ii) $V_{i} / V_{i+1}$ (with the canonical parabolic structure) is parabolic stable, and

(iii) $\mathrm{gr} V=\bigoplus V_{i} / V_{i+1}$ is well determined, i.e., gr $V$ (up to parabolic isomorphism) is independent of the filtration $\left\{V_{i}\right\}$ of $V$ with properties (i) and (ii).

Let $V B(d, \alpha)$ denote the category of parabolic semistable vector bundles $V$ on $X$ with a parabolic structure at a single point $Q \in X$ (we assume this for simplicity of notation) of fixed weight $\alpha=\left(\alpha_{1}, \ldots, \alpha_{n}\right)$ and fixed ordinary degree $d$. Let $\sim$ denote the equivalence relation in $V B(d, \alpha), V_{1} \sim V_{2}$ if $\mathrm{gr} V_{1}$ $=\operatorname{gr} V_{2}$. Let $M(d, \alpha)$ be the set of equivalence classes under this equivalence relation. 
THEOREM 1. Suppose that $g=$ genus of $X \geqslant 2$. Then there is a natural structure of a normal projective variety on $M(d, \alpha)$ of dimension $n^{2}(g-1)+\delta$ where $\delta$ is the dimension of the variety of flags in an n-dimensional vector space of type given by the type of the underlying quasi-parabolic structure. Further $M(d, \alpha)$ is smooth at the points $V$ where $V$ is parabolic stable.

Suppose now that the base field $k=\mathrm{C}$ and $X-Q=H \bmod \Gamma$ where $H$ is the upper half plane and $\Gamma$ is a discrete subgroup of Aut $H$. Fix a parabolic fixed point $Q^{\prime}, Q^{\prime} \in \bar{H}$ of $\Gamma(\bar{H}$ being the usual $H \cup$ certain boundary points). Let $\Gamma_{0}$ be the isotropy subgroup of $\Gamma$ at $Q^{\prime}$. Fix a generator $\gamma_{0}$ of $\Gamma_{0}\left(\Gamma_{0} \approx Z\right)$. Let $R(\alpha)$ denote the equivalence classes of unitary representations $\chi$ of $\Gamma$ such that $\chi\left(\gamma_{0}\right)$ is conjugate to the diagonal matrix with entries $\left(e^{2 \pi i \alpha_{1}}, \ldots, e^{2 \pi i \alpha_{n}}\right)$, $\alpha=\left(\alpha_{1}, \ldots, \alpha_{n}\right), 0 \leqslant \alpha_{1} \leqslant \alpha_{2} \cdots \leqslant \alpha_{n}<1$.

THEOREM 2. Suppose that $g \geqslant 2$. Then there is a canonical identification of $R(\alpha)$ with the underlying topological space of $M(d, \alpha)$ with $d=-\Sigma_{i=1}^{n} \alpha_{i}$ (or equivalently par $\operatorname{deg} V=0, V \in M(d, \alpha)$ ).

\section{REFERENCES}

1. D. Mumford, Projective invariants of projective structures and applications, Proc. Internat. Congr. Mathematicians (Stockholm, 1962), Inst. Mittag-Leffler, Djursholm, 1963, pp. 526-530. MR 31 \# 175 .

2. - Geometric invariant theory, Academic Press, New York; Springer-Verlag, Berlin, 1965. MR 35 \# 5451.

3. M. S. Narasimhan and C. S. Seshadri, Stable and unitary vector bundles on a compact Riemann surface, Ann. of Math. (2) 85 (1965), 540-567. MR 32 \# 1725.

4. C. S. Seshadri, Space of unitary vector bundles on a compact Riemann surface, Ann. of Math. (2) 85 (1967), 303-336. MR 38 \# 1693.

5. - Moduli of $\pi$-vector bundles on an algebraic curve, Questions on Algebraic Varieties (C.I.M.E., III Ciclo, Varenna, 1969), Edizioni Cremonese, Rome, 1970, pp. 139260. MR 43 \# 6216.

6. A. Weil, Généralisation des fonctions abéliennes, J. Math. Pures Appl. 17 (1938), 47-87.

TATA INSTITUTE OF FUNDAMENTAL RESEARCH, BOMBAY, INDIA

INSTITUTE FOR ADVANCED STUDY, SCHOOL OF MATHEMATICS, PRINCETON, NEW JERSEY 08540 